\title{
Two cases of malignant glaucoma unresolved by pars plana vitrectomy
}

This article was published in the following Dove Press journal:

Clinical Ophthalmology

02 April 2014

Number of times this article has been viewed

\section{Yoshikatsu Hosoda \\ Tadamichi Akagi \\ Nagahisa Yoshimura}

Department of Ophthalmology and Visual Sciences, Kyoto University Graduate School of Medicine, Kyoto, Japan
Correspondence:Tadamichi Akagi Department of Ophthalmology and Visual Sciences, Kyoto University Graduate School of Medicine, 54 Shogoin Kawahara-cho, Sakyo-ku, Kyoto 606-8507, Japan

$\mathrm{Tel}+8 \mid 7575$ I 3250

Fax +81757520933

Email akagi@kuhp.kyoto-u.ac.jp
Abstract: Malignant glaucoma, which is characterized by a shallow or flat anterior chamber with high intraocular pressure, can usually be resolved by pars plana vitrectomy with anterior hyaloidectomy. We describe two cases in which malignant glaucoma was refractory to conventional treatment and complete vitrectomy. Case one an 88-year-old woman with pseudoexfoliation glaucoma underwent trabeculotomy and subsequently developed malignant glaucoma. Four months after transient recovery by pars plana vitrectomy, the malignant glaucoma recurred. She underwent peripheral iridectomy and local zonulectomy with successful control of her intraocular pressure. In case two, an 85-year-old man had a history of pseudoexfoliation glaucoma. Seven months after phacoemulsification and intraocular lens implantation, he developed malignant glaucoma that was refractory to pars plana vitrectomy. He underwent peripheral iridectomy, goniosynechialysis and trabectome surgery resulting in the successful control of his intraocular pressure. In rare cases of malignant glaucoma refractive to vitrectomy, peripheral iridectomy with or without local zonulectomy is a reasonable and minimally invasive surgical procedure.

Keywords: malignant glaucoma, pars plana vitrectomy, peripheral iridectomy

\section{Introduction}

Malignant glaucoma was first described by Von Graefe in $1869 .{ }^{1}$ It is a rare postoperative complication characterized by a flattening of the anterior chamber and elevated intraocular pressure (IOP), and is also known as ciliary block glaucoma or aqueous misdirection. Various medical, laser-based, and surgical treatments for malignant glaucoma have been reported in the literature. ${ }^{2}$ Although medical treatment such as topical cycloplegics, topical aqueous suppressants, oral carbonic anhydrase inhibitors, and systemic hyperosmotic agents, should be considered first, it is not effective solely in approximately $50 \%$ of malignant glaucoma cases. ${ }^{3}$ Other treatment options include neodymium-doped yttrium aluminum garnet (Nd:YAG) laser capsulohyaloidotomy or surgical disruption of the anterior hyaloid. In cases that are refractory to the aforementioned treatment options, pars plana vitrectomy (PPV) with or without lensectomy can be used. ${ }^{4}$ Diode laser cyclophotocoagulation would be another option in cases that are refractory to all other treatments.

Here, we report two rare cases in which malignant glaucoma was refractory to conventional treatment and complete vitrectomy. At 4 and 1 month, respectively, after PPV, malignant glaucoma recurred and the patients underwent peripheral iridectomy which resulted in successful control of their IOP.

\section{Case reports}

Case one was an 88-year-old woman with a history of pseudoexfoliation glaucoma in the right eye (OD) with maximum IOP $>30 \mathrm{mmHg}$. Both eyes were pseudophakic and showed normal anterior chamber depth. Because her IOP OD could not be controlled 
with additional glaucoma eyedrops and oral acetazolamide, she underwent trabeculotomy. Three days after the trabeculotomy, she developed a flattening of the anterior chamber and an elevated IOP OD. She was diagnosed with malignant glaucoma. The condition could not be resolved with systemic hyperosmotic agents and oral carbonic anhydrase inhibitors, so she underwent an emergent PPV (total vitrectomy) using a 25 gauge vitreous cutter with vitreous base shaving and removal of the anterior hyaloid. Her anterior chamber deepened, and her IOP decreased to $10 \mathrm{mmHg}$ the following day. Her IOP was maintained in the mid-teens OD by using three types of glaucoma eyedrops. Four months after PPV, her IOP increased to $70 \mathrm{mmHg}$ again with a flat anterior chamber (Figure 1A, B). We hypothesize that the cilio-pupillary block mechanism was related to her clinical condition, and we performed an emergent iridectomy and local zonulectomy using a 25 gauge vitreous cutter. The posterior synechia of the iris was partially, but not completely, observed intraoperatively. Her anterior chamber had deepened (Figure 1C, D); for 10 months, her IOP has been maintained in the low-teens OD with the use of two types of glaucoma eyedrops.

Case two was an 85-year-old man with a history of primary angle closure in both eyes (OU) and pseudoexfoliation glaucoma OD. He underwent phacoemulsification and aspiration (PEA), and intraocular lens (IOL) implantation OU. Following cataract surgery, he frequently exhibited a flattening of the anterior chamber and elevated IOP of $>40 \mathrm{mmHg}$ on maximal glaucoma medications. As his clinical condition OD was considered to be related to the

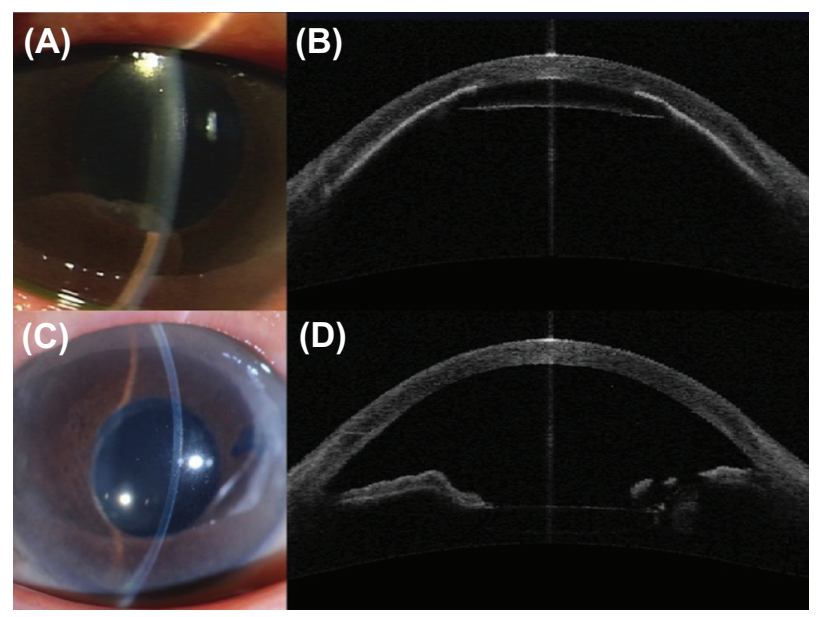

Figure I An 88-year-old woman presented with a recurrence of malignant glaucoma in her right eye 4 months after pars plana vitrectomy.

Notes: Slit lamp photograph (A), and anterior segment optical coherence tomography image (B) before peripheral iridectomy and local zonulectomy. The anterior chamber disappeared, and the iris was thoroughly in contact with the corneal endothelium. Slit lamp photograph (C), and anterior segment optical coherence tomography image (D), 2 months after peripheral iridectomy and local zonulectomy. The anterior chamber had deepened significantly.

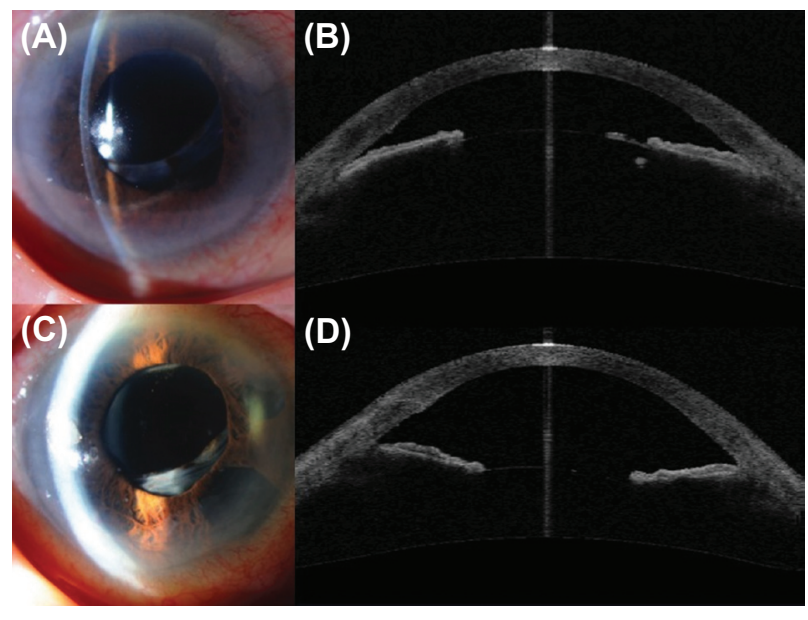

Figure 2 An 85-year-old man presented with a recurrence of malignant glaucoma in his right eye I month after pars plana vitrectomy.

Notes: Slit lamp photograph (A), and anterior segment optical coherence tomography image (B) showed a flattening of the anterior chamber and a dislocated intraocular lens. The central anterior chamber depth was $1.49 \mathrm{~mm}$. Slit lamp photograph (C), and anterior segment optical coherence tomography image (D), revealed that the anterior chamber depth had increased to $2.97 \mathrm{~mm}$ at the center after patent iridectomy with goniosynechialysis and trabectome surgery.

malignant glaucoma mechanism, he underwent a YAG capsulohyaloidotomy and goniosynechialysis (GSL) for severe peripheral anterior synechia (PAS). Ten months after cataract surgery, his IOP OD was $32 \mathrm{mmHg}$, and his anterior chamber was still very shallow. He then underwent PPV (total vitrectomy) using a 25 gauge vitreous cutter with vitreous base shaving and removal of the anterior hyaloid, and GSL OD, at which point his anterior chamber deepened significantly and his IOP decreased to the low-teens. One month after PPV, he once again exhibited a flattening of the anterior chamber and severe PAS, and was referred to Kyoto University Hospital (Figure 2A, B). He presented with spontaneous IOL dislocation within the capsular bag. His IOP OD increased to $32 \mathrm{mmHg}$, and his visual acuity worsened to counting finger vision. He underwent an iridectomy without zonulectomy using a 25 gauge vitreous cutter near the area where Zinn's zonule had been partially ruptured. We further performed GSL and trabectome surgery (NeoMedix Corporation, Tustin, CA, USA) (a surgical procedure that ablates an arc of trabecular meshwork). His anterior chamber had deepened (Figure 2C, D); for 6 months, his IOP has been maintained in the high-teens OD by using additional glaucoma eyedrops.

\section{Discussion}

PPV has been considered to be efficacious in the treatment of pseudophakic malignant glaucoma. ${ }^{5}$ It is important to perform total vitrectomy with surgical disruption of the anterior hyaloid to break the primary mechanism of aqueous misdirection. 
However, it was previously reported that patients experienced recurrence of malignant glaucoma following vitrectomy, which is also called malignant glaucoma-like syndrome. ${ }^{6,7}$ In such cases, the flattening of anterior chamber spaces and the elevation of IOP are caused by hydration of the vitreous cavity, not the vitreous. Our two cases showed the ciliopupillary block caused by the forward movement of the IOL due to a weak or partially ruptured zonular apparatus and dislocated IOL. It has been shown ${ }^{8}$ that malignant glaucoma is more common in females due to the lens being in a more forward position, and shallower anterior chamber, although, in our cases, one patient was female and the other male. Our cases both presented with pseudoexfoliation syndrome, which could be a risk factor for malignant glaucoma due to its weak zonule and possibility of rupture. In case one, partial posterior synechia of the iris was observed during surgery; thus, it is possible that partial posterior synechia and anterior dislocated IOL caused the cilio-pupillary block. As the patient's PAS was not severe, the IOP could be controlled adequately with peripheral iridectomy and local zonulectomy alone. In case two, total posterior synechia of the iris was identified intraoperatively, and the cilio-pupillary block caused by the posterior synechia of the iris was presumed to have caused the elevated IOP. Because peripheral iridectomy alone seemed to be insufficient to reduce the IOP for over $90 \%$ PAS of the angle circumference, the patient underwent GSL, trabectome surgery, and patent peripheral iridectomy. The benefit of trabectome surgery is its availability in the operative field, similar to GSL, and its efficacy in treating possible functional disorders of the trabecular meshwork. In case two, local zonulectomy was not performed because the Zinn's zonule had been partially ruptured.

\section{Conclusion}

Our two cases demonstrate a recurrence of malignant glaucoma following PPV. It has been reported that hyaloid-zonulectomy and iridectomy are effective in treating aqueous misdirection. ${ }^{9}$ We also suggest that patent peripheral iridectomy with, or without local zonulectomy is an effective surgical procedure in the prevention of prospective ciliary, or pupillary blockage, regardless of whether the patient has previously undergone any type of vitrectomy to treat malignant glaucoma in cases of a weak zonular apparatus, dislocated IOL, or severe posterior synechia. In cases of severe PAS, a combined procedure, including the reconstruction of the trabecular meshwork with such procedures as GSL and trabectome surgery, may also be an effective treatment, as we demonstrated. In conclusion, peripheral iridectomy is a appropriate and minimally invasive surgical procedure used to break the cycle of malignant glaucoma refractive to conventional treatments and PPV.

\section{Disclosure}

The authors report no conflicts of interest in this work.

\section{References}

1. Von Graefe A. Beiträge zur pathologie und therapie des glaucoms [Contributions to the pathology and therapy of glaucoma]. Archiv Für Ophthalmologie. 1869;15(3):108-252. German.

2. Dave P, Senthil S, Rao HL, Garudadri CS.Treatment outcomes in malignant glaucoma. Ophthalmology. 2013;120(5):984-990.

3. Ng WT, Morgan W. Mechanisms and treatment of primary angle closure: a review. Clin Experiment Ophthalmol. 2012;40(4):e218-e228.

4. Harbour JW, Rubsamen PE, Palmberg P. Pars plana vitrectomy in the management of phakic and pseudophakic malignant glaucoma. Arch Ophthalmol. 1996;114(9):1073-1078.

5. Lynch MG, Brown RH, Michels RG, Pollack IP, Stark WJ. Surgical vitrectomy for pseudophakic malignant glaucoma. Am J Ophthalmol. 1986;102(2):149-153.

6. Massicotte EC, Schuman JS. A malignant glaucoma-like syndrome following pars plana vitrectomy. Ophthalmology. 1999;106(7):1375-1379.

7. Song J, Castellarin A, Song M, Song A. Repositioning of glaucoma tubes into the pars plana for refractory malignant glaucoma: a case report. $J$ Med Case Rep. 2013;7(1):102.

8. Razeghinejad MR, Amini H, Esfandiari H. Lesser anterior chamber dimensions in women may be a predisposing factor for malignant glaucoma. Med Hypotheses. 2005;64(3):572-574.

9. Bitrian E, Caprioli J. Pars plana anterior vitrectomy, hyaloidozonulectomy, and iridectomy for aqueous humor misdirection. $\mathrm{Am}$ J Ophthalmol. 2010;150(1):82-87.
Clinical Ophthalmology

\section{Publish your work in this journal}

Clinical Ophthalmology is an international, peer-reviewed journal covering all subspecialties within ophthalmology. Key topics include: Optometry; Visual science; Pharmacology and drug therapy in eye diseases; Basic Sciences; Primary and Secondary eye care; Patient Safety and Quality of Care Improvements. This journal is indexed on

\section{Dovepress}

PubMed Central and CAS, and is the official journal of The Society of Clinical Ophthalmology (SCO). The manuscript management system is completely online and includes a very quick and fair peer-review system, which is all easy to use. Visit http://www.dovepress.com/ testimonials.php to read real quotes from published authors. 